\title{
Effects of GLP-1 Analogue, Liraglutide on the Expression of Mouse Pre-osteoblast MC3T3-E1
}

\author{
ZHIHUI GAO, SHUAI ZHANG, LONG PANG, PENG LI, XIWU GAO AND QUNHUA JIN* \\ Third Department of Orthopaedics, General Hospital of Ningxia Medical University, No. 804 South Shengli Street, Xingqing \\ District, Yinchuan City, Ningxia Hui Autonomous Region, 750004, China
}

\section{Gao et al.: Effect of Liraglutide on Mouse Pre-osteoblast MC3T3-E1 expression}

\begin{abstract}
This investigation dealt with the investigation of the effects of GLP-1 analogue, liraglutide on the expression of mouse pre-osteoblast MC3T3-E1, MC3T3-E1 cells were cultured, passaged and frozen. Cell lines were cultured under different conditions and were separated into control group exposed to saline and groups 2, 3 and 4, which were exposed to $10^{-9} \mathrm{M}, 10^{-8} \mathrm{M}$ and $10^{-7} \mathrm{M}$ liraglutide, respectively. Group 2 cells after 2 d of intervention were subjected to CCK-8 assay for cell proliferation, flow cytometry for cell apoptosis and Western blot for protein expression. The expression of osteoblast differentiation marker genes, GLP1R, Runx2, OCN and ALP were detected using real-time fluorescence quantitative PCR 3, 6, 12 and 20 days after intervention. The results showed that liraglutide promoted proliferation of osteoblasts and the reduced early apoptotic rate of osteoblasts, which indicated that liraglutide could promote the proliferation and differentiation of mouse osteoblasts MC3T3-E1 and inhibited the apoptosis of cells.
\end{abstract}

Key words: GLP-1, liraglutide, osteoblasts, MC3T3-E1

Diabetes mellitus (DM) is a common endocrine system disease characterized by disorders of glucose metabolism, which is based on the interaction of genetic factors, dietary factors or environmental factors ${ }^{[1,2]}$. DM can cause various secondary complications such as damage to blood vessels or organs, have a great impact on the daily life of patients, and increase the difficulty of life of patients ${ }^{[3,4]}$. Osteoporosis (OP) is a metabolic disease which is prone to fracture because of the decrease of bone mass and the increase of bone fragility. Studies have found that type 2 DM (T2DM) can lead to bone loss and reduction of bone mineral density. T2DM can lead to bone instability and increased incidence of fractures in patients ${ }^{[5-8]}$. It is now considered that OP is a chronic complication of DM. Bone mineral density of diabetic patients will be affected by insulin resistance, hyperglycemia, estrogen and other factors. In elderly patients, the incidence of OP and or T2DM is increasing year by year ${ }^{[9,10]}$. These two diseases can lead to an increase in the rate of fractures, which can lead to vascular complications or disabilities. With the increase of the incidence of osteoporotic fracture, the mortality rate increases and the quality of life decreases.
Current studies have reported that GLP-1 is not only related to glucose and lipid metabolism, but also indispensable for bone remodeling ${ }^{[11]}$. Osteoblasts (OB) are mainly mesenchymal progenitor cells derived from periosteum and bone marrow stroma. Their main function is to stabilize bone homeostasis. OBs regulate the production of extracellular matrix proteins, matrix mineralization, osteoclast differentiation, and bone remodeling ${ }^{[12,13]}$. It has a great influence on the pathogenesis of many skeletal diseases, especially OP. Over the years, the main method of treating OP is to inhibit the maturation, proliferation and activity of osteoclasts against bone resorption ${ }^{[14]}$. In recent years, the use of metabolic therapy for OP has attracted a lot of attention. It has been found that metabolic drugs can upregulate $\mathrm{OB}$ precursors, accelerate the differentiation of precursor cells into mature OBs and increase the function and activity of mature OBs, thereby upregulating the synthesis of bone matrix and bone formation ${ }^{[15]}$. In view of the interaction between $\mathrm{DM}$ and $\mathrm{OP}$, the effect of antidiabetic drugs on bone metabolism has become the new research focus in the field of OP. 
Nowadays, few results have been obtained about the effect of GLP-1 on osteoblasts. In this study, the effects of GLP-1 analogue, liraglutide on the proliferation, differentiation and apoptosis of mouse osteoblast MC3T3-E1 cells were studied through detecting the expression of osteoblast differentiation markers, GLP1R, Runx2, OCN and ALP, and the expression of apoptosis-related Bax and Bcl-2. It is hoped that this study could provide a theoretical basis for patients with $\mathrm{DM}$ and OP to choose hypoglycemic drugs.

\section{MATERIALS AND METHODS}

\section{Cell line and cell culture:}

Pre-osteoblast-like cells MC3T3-E1 were obtained from Wuxi Innovate Biomedical Technology Co., Ltd., China. Thirty minutes before the start of the experiment the cell culture bottles (Wuxi NEST Biotechnology Co., Ltd., China) were taken out from the incubator, culture bottle was opened under sterile conditions and the old culture medium was aspirated out. The cells were washed $3 \times$ with $2 \mathrm{ml}$ of PBS buffer (Hyclone Company, USA) and replaced with fresh and complete culture medium, the bottle is placed in the incubator, and the culture medium was replaced every 2-3 days. The changes of color and transparency of culture medium were observed every day along with the morphology, density and adherence of MC3T3-E1 cells was observed under inverted microscope.

\section{Cell passage:}

When the degree of cell fusion in the culture flask reached $90 \%$, the cells were subcultured. About $1 \mathrm{ml}$ of buffer containing $0.02 \%$ EDTA and $0.25 \%$ trypsin digestate (Beijing Solarbio Science and Technology Co., Ltd., China) was added, shaken for $60 \mathrm{~s}$ and observed under a microscope. The enlargement of intercellular space, retraction and roundness of cells were observed, and the digestion was terminated by adding full medium of equal volume. Complete detachment of the cells from the bottle wall was ensured and the cell suspension was transferred to a $15 \mathrm{ml}$ centrifuge tube (Wuxi NEST Biotechnology Co., Ltd., China) and centrifuged at $1000 \mathrm{rpm}$ for $4 \mathrm{~min}$. The supernatant was discarded and an appropriate amount of complete medium was added to make a uniform cell suspension.

\section{Cell cryopreservation:}

MC3T3-E1 cells in the log phase and trypsin-digested adherent cells were selected. The cell suspension was centrifuged at $1000 \mathrm{rpm}$ for $4 \mathrm{~min}$, the supernatant was aspirated and cell cryopreservation solution was added. The cell concentration was adjusted to $1 \times 10^{5}$ $-2 \times 10^{6} / \mathrm{ml}$. The uniform cell suspension was transferred to a cryotube (Wuxi NEST Biotechnology Co., Ltd., China), with 1-1.5 ml per tube sealed and labeled with time and quantity.

\section{Grouping:}

Mouse pre-osteoblast MC3T3-E1 cells were cultured in complete culture medium (each $100 \mathrm{ml}$ contained $89 \mathrm{ml} \alpha$-MEM medium, $10 \mathrm{ml} \mathrm{10 \%} \mathrm{fetal} \mathrm{bovine} \mathrm{serum}$

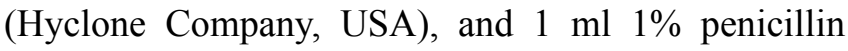
double antibody (Beijing Solarbio Science and Technology Co., Ltd., China). When the cell growth reached log phase, the cells were used. The cells were divided into 4 groups. The control group and it was treated with equal volume of saline. The remaining 3 groups were the intervention groups and were exposed to different concentrations of liraglutide (Novo Nordisk Company, Denmark). Group 2 cells were exposed to $10^{-9} \mathrm{M}$ liraglutide, group 3 cells to $10^{-8} \mathrm{M}$ and group 4 cells to $10^{-7} \mathrm{M}$ liraglutide. Each group in the intervention group were exposed for $48 \mathrm{~h}$.

\section{CCK-8 assay for cell proliferation:}

After digesting the adherent cells, the cells were counted, the cell concentration is adjusted to 6000 cells/ $\mu 1$ and the cell suspension was added to a 96well plate, about $100 \mu$ of cell suspension was seeded into each well, $100 \mu \mathrm{l}$ of PBS is added to the edge well, and then the 96-well plate is placed in an incubator and cultivated. After the cells adhered completely, the old medium was discarded and the whole medium was incubated with $0,10^{-9}, 10^{-8}$ and $10^{-7} \mathrm{M}$ liraglutide. The plates were placed in an incubator and cultivated for $48 \mathrm{~h}$. After completion of the exposure, $10 \mu \mathrm{l}$ of CCK8 reagent (Shanghai BestBio Biological Company, China) was added to each well, incubated for $4 \mathrm{~h}$, and the absorbance of each well was measured at $450 \mathrm{~nm}$ in a microplate reader. Percent cell proliferation was calculated using the formula, $\%$ cell proliferation $=$ (OD of treated cells-OD of blank)-(OD of control cellsOD of blank $) /($ OD of control cells-OD of blank $) \times 100$

\section{Flow cytometry to detect apoptosis and cell cycle:}

Cells were digested with $0.25 \%$ trypsin. The treated cell suspension ( $3 \mathrm{ml}$ per well) was inoculated into a 6-well plate. Three parallel control groups were set up in each group. The 6-well plates were further cultured and when the cells adhered to the wall the old culture 
medium was discarded. Complete culture medium with concentration of $0,10^{-9}, 10^{-8}$ and $10^{-7} \mathrm{M}$ liraglutide was added to the 6-well plate. The plate was incubated for $48 \mathrm{~h}$ and adherent cells digested with $0.25 \%$ trypsin. After digestion, complete medium was added to avoid over-digestion. The cell suspension was transferred to the centrifugal tube and centrifuged at $1000 \mathrm{rpm}$ for $4 \mathrm{~min}$. The supernatant was discarded, the pellet was washed with $2 \mathrm{ml}$ PBS twice. After washing, the cells were centrifuged at $2000 \mathrm{rpm}$ for $4 \mathrm{~min}$ and the supernatant was discarded. The cells were suspended in $500 \mu \mathrm{l}$ of binding buffer, $5 \mu \mathrm{L}$ FITC labeled Annexin V solution was added, mixed, $5 \mu$ PI (Nanjing KeyGen Biotech. Inc., China) was added in the dark, mixed evenly, kept for $15 \mathrm{~min}$, at room temperature. Flow cytometry was performed within $1 \mathrm{~h}$ keeping the emission wavelength at $530 \mathrm{~nm}(\mathrm{Em})$ and the excitation wavelength at $488 \mathrm{~nm}$ (Ex). The expression of osteoblast differentiation markers GLP-1R, Runx2, OCN and ALP genes were detected using real-time fluorescence quantitative PCR, 3, 6, 12 and $20 \mathrm{~d}$ after liraglutide exposure.

\section{Total RNA extraction:}

Cells were inoculated into $25 \mathrm{ml}$ cell culture flask and cultured for $48 \mathrm{~h}$ after cell adherence. After discarding the old culture medium, $1 \mathrm{ml}$ Trizol (Invitrogen Company, USA) was added to each cell culture bottle, mixed and placed at room temperature for $5 \mathrm{~min}$, so that the protein ribozymes are completely separated. It was then transferred to a $1.5 \mathrm{ml}$ EP tube, $0.2 \mathrm{ml}$ chloroform was and shaken for $15 \mathrm{~s}$. It was placed on ice for $3 \mathrm{~min}$. centrifuged in a refrigerated high-speed centrifuge (Eppendorf) at $12000 \mathrm{rpm}$ for $15 \mathrm{~min}$. After centrifugation, the sample separated into 3 layers. RNA in the upper colorless aqueous phase is transferred to a new $1.5 \mathrm{ml} \mathrm{EP}$ tube. Isopropanol of equal volume was added to the EP tube, mixed, and kept aside for $10 \mathrm{~min}$. Centrifugation at $4^{\circ}, 12000 \mathrm{rpm}$ for $10 \mathrm{~min}$. The supernatant was carefully aspirated without disturbing the RNA deposits at the bottom of the tube. The EP tube was washed with $75 \%$ ethanol for RNA precipitation, centrifuged at $4^{\circ}, 7500 \mathrm{rpm}$ for $3 \mathrm{~min}$, supernatant was aspirated out without disturbing the RNA pellet, which was dried, $20 \mu 1$ DEPC water (Beijing Solarbio Science and Technology Co., Ltd., China) was added to dissolve the RNA and stored at $-80^{\circ}$.

\section{RNA purity and concentration detection:}

OD values of RNA at 260 and $280 \mathrm{~nm}$ were measured on a NanoDrop 2000 Microspectrophotometer (Thermo
Scientific Company, USA). The purity of RNA was determined by calculation the ratio OD260/OD280, and a ratio between 1.8 and 2.0 indicated that the RNA was of high purity.

\section{Reverse transcription reaction to synthesize DNA:}

Frozen RNA was thawed for $5 \mathrm{~min}$ in a $65^{\circ}$ water bath, then cooled immediately on ice. The reaction liquid was gently mixed, centrifuged in a microcentrifuge for $30 \mathrm{~s}$, placed on ice, reacted at $37^{\circ}$ for $15 \mathrm{~min}, 50^{\circ}$ for $5 \mathrm{~min}$ and at $98^{\circ}$ for $5 \mathrm{~min}$, cooled at $4^{\circ}$ until stable and stored at $-20^{\circ}$.

\section{RT-PCR reaction:}

Under dark, the reaction solution was prepared in the fluorescent quantitative EP tube according to SYBR Green Realtime PCR Master Mix instructions, centrifuged for $40 \mathrm{~s}$ at $1000 \mathrm{rpm}$, and placed in the fluorescent quantitative PCR instrument. Forty denaturation-annealing-elongation cycles were performed and it was analyzed using Mxpro software.

\section{Western blot:}

SDS-PAGE gel electrophoresis was carried out by adding marker and protein samples and electrophoresis was carried out until bromophenol blue moved to the bottom of the gel. After taking out the gel, the target protein gel was cut according to the size of protein marker and then immersed in the electroplating fluid. According to the size of gel, the PVDF membrane is cut (Beijing Biosynthesis Biotechnology co., Ltd., China). It was immersed in methanol for $15 \mathrm{~s}$ and immersed in the electrolyte for $15 \mathrm{~min}$. The sandwich structure was made from the bottom up filter paper, PVDF film, gel and filter paper. The bubbles were removed between each layer, the electrodes are connected, and the power supply is connected. According to the size of the PVDF film and the size of the target protein, the transmembrane pressure and the film transfer time were chosen.

\section{Immuno-hybridization and development:}

PVDF membranes were sealed for $1-2 \mathrm{~h}$ with $5 \%$ milk sealing solution at $37^{\circ}$. The PVDF membrane was incubated with an antidilution solution overnight at $4^{\circ}$. The PVDF membrane was removed and washed $3 \times$ with TBST for 15 min each time. PVDF membranes were incubated at $37^{\circ}$ for $1 \mathrm{~h}$ with the diluent of the second antibody prepared with the enclosed solution. 
The PVDF membrane was removed and washed $3 \times$ with TBST for 15 min each time. The luminescent liquid is placed in the gel imager, and the optical density of the target strip is analyzed.

\section{Statistical analysis:}

SPSS16.0 software is used for statistical analysis. All the experiments are repeated 3 times, and the quantitative results are expressed by the mean \pm standard deviation (SD). Independent sample $\mathrm{T}$ test is used to compare the quantitative values between the two groups. Single factor analysis of variance is used to compare the quantitative values between the 2 groups, and $\mathrm{S}-\mathrm{N}-\mathrm{K}$ method was used to compare the quantitative values between the 2 groups. $\mathrm{P}<0.05$ was regarded as significant difference.

\section{RESULTS AND DISCUSSION}

Compared to the control group, the proliferation rate of osteoblasts in the intervention group was significantly higher $(\mathrm{P}<0.05)$. The effect of $10^{-8} \mathrm{M}$ liraglutide on osteoblast proliferation was the most significant as shown in fig. 1. Compared to the control group, the expression of GLP-1R, Runx 2 and OCN in the liraglutide intervention group increased significantly $(\mathrm{P}<0.05)$ after 3, 6, 12 and $20 \mathrm{~d}$ of exposure to liraglutide $\left(10^{-9}, 10^{-8}\right.$ and $\left.10^{-7} \mathrm{M}\right)$, and the expression of GLP-1R, Runx2 and OCN increased significantly in the $10^{-7} \mathrm{M}$ treated group (fig. 2). Compared to the control group, the activity of ALP in the $10^{-7} \mathrm{M}$ liraglutide treated group increased significantly as shown in fig. 3 .

Compared to the control group, the expression of apoptotic protein Bax in the liraglutide intervention
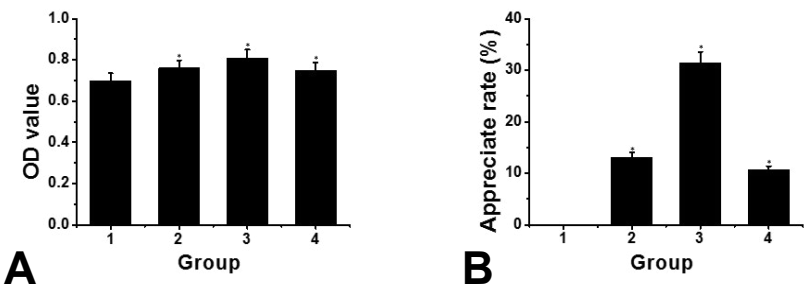

Fig. 1: Effects of liraglutide on proliferation of osteoblasts MC3T3-E1

Effects of different concentrations of liraglutide on proliferation of osteoblasts MC3T3-E1. A. Comparison of OD values among the 4 groups, B. comparison of proliferation rates among the 4 groups, $\left.{ }^{*} \mathbf{P}<0.05\right)$.
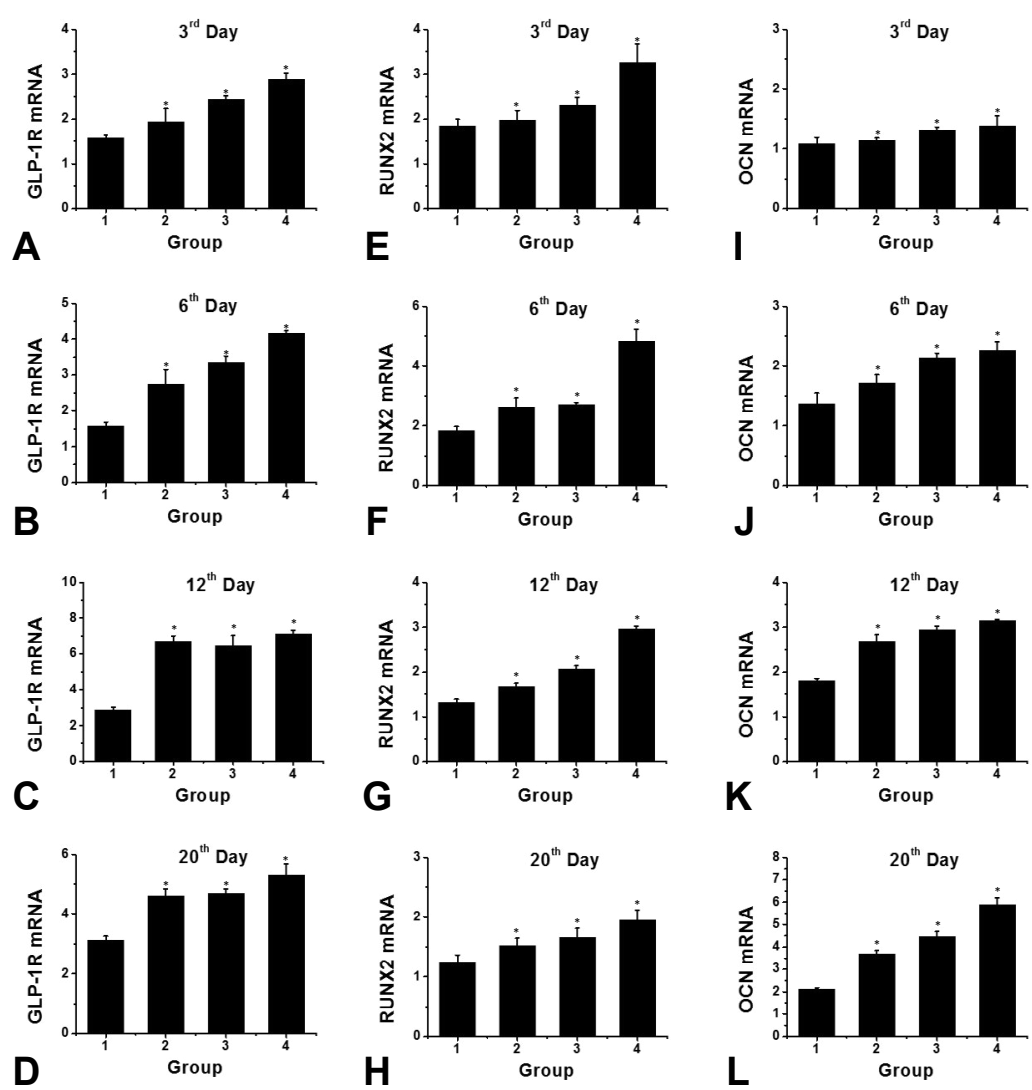

Fig. 2: Comparative expression of GLP-1R, Runx2 and OCN in different groups on different days Comparison of the expression of GLP-1R, Runx 2 and OCN in different groups. A-D. GLP-1R expression in each group at 3, 6, 12 and 20 days, E-H. RUNX2 expression in each group at 3, 6, 12 and 20 days and I-L. OCN expression in each group at 3, 6, 12 and 20 days. 

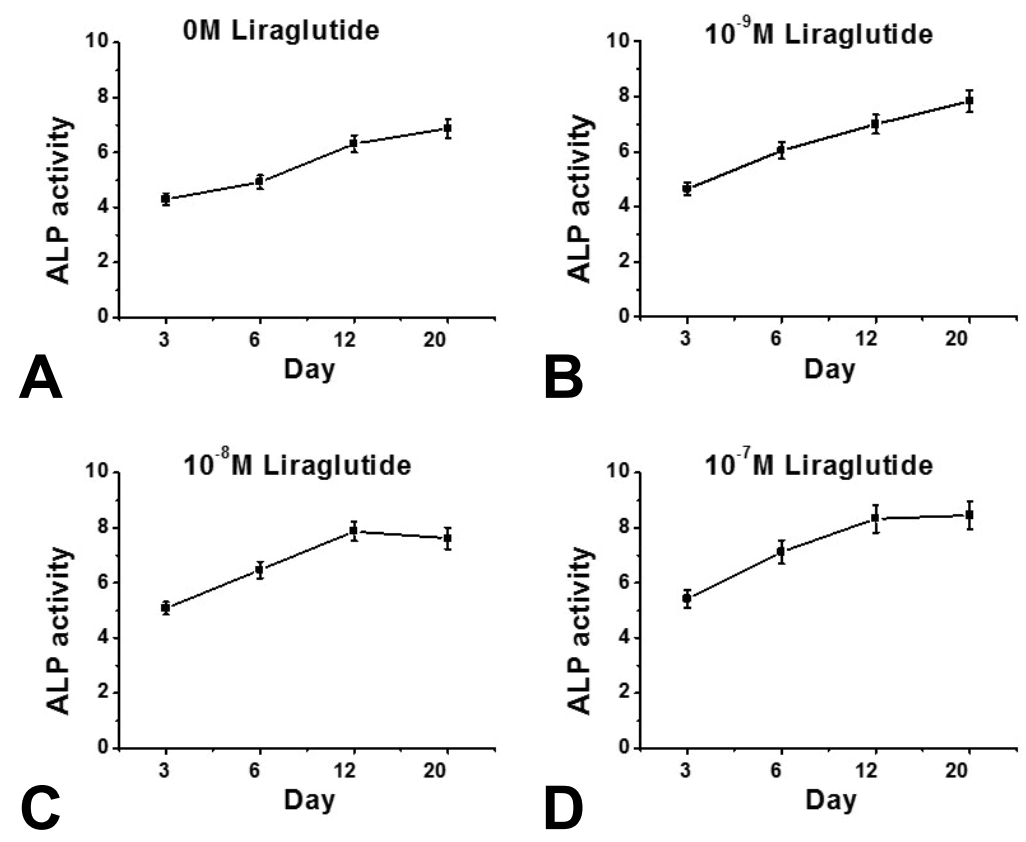

Fig. 3: ALP activity in each group after exposure to liraglutide

Changes of ALP activity in each group after exposure to liraglutide, A. ALP activity in control group at 3, 6, 12 and 20 days, B. ALP activity in $10^{-9} \mathrm{M}$ intervention group 2 at 3, 6, 12 and 20 days $\mathrm{C}$. Changes of ALP activity in $10^{-8} \mathrm{M}$ intervention group 3 at 3, 6, 12 and 20 days, and $D$. ALP activity in $10^{-7} M$ intervention group 4 at 3, 6, 12 and 20 days.
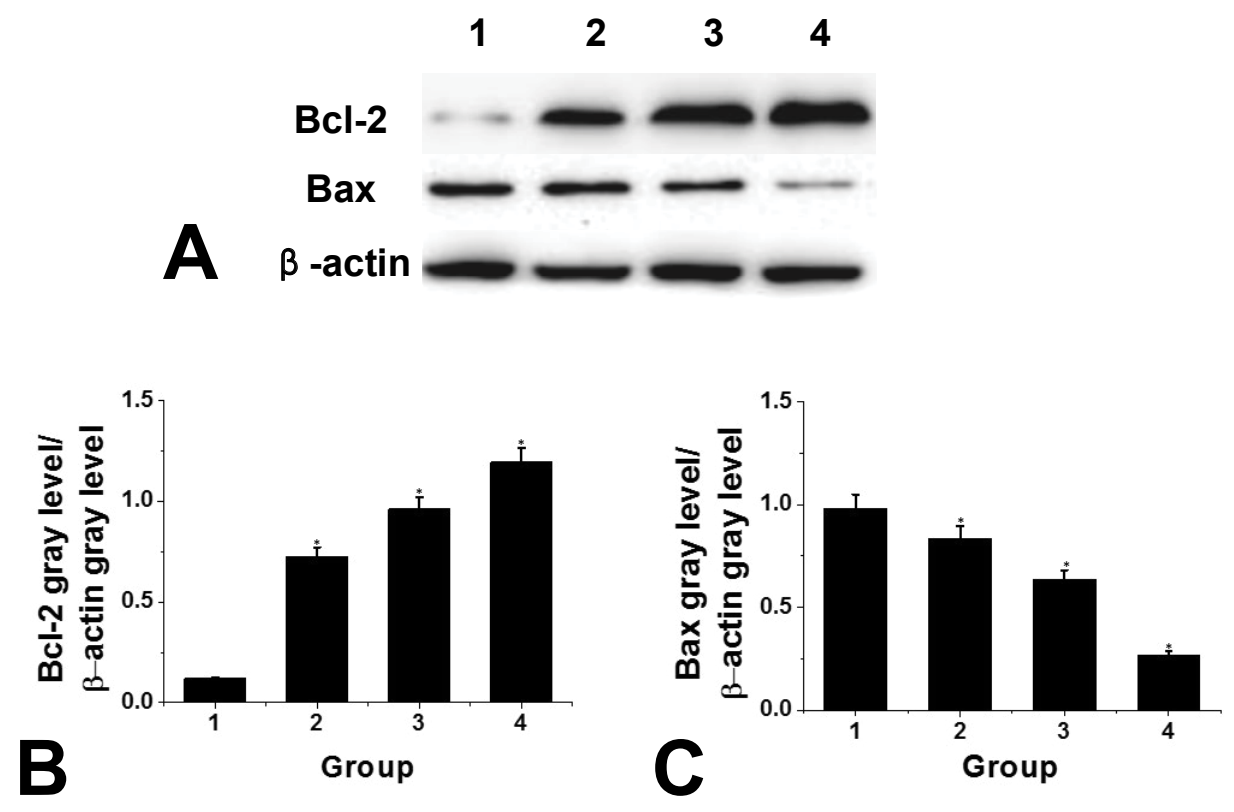

Fig. 4: Effect of liraglutide on apoptotic protein expression

Effect of liraglutide on apoptotic protein expression, A. electrophoregram of Bcl-2 and Bax expression in osteoblasts after $48 \mathrm{~h}$ of liraglutide intervention, B. comparison of Bcl-2 expression in osteoblasts after intervention in each group, C. comparison of Bax expression in osteoblasts after intervention in each group, ${ }^{*} \mathrm{P}<0.05$

group is significantly lower $(\mathrm{P}<0.05)$ and with increasing concentration of liraglutide, the expression of Bax showed a decreasing trend. There is a significant difference between the 2 groups. Compared to the control group, the expression of $\mathrm{Bcl}-2$ protein in liraglutide intervention group increased significantly $(\mathrm{P}<0.05)$ and with increasing liraglutide concentration, the expression of Bcl-2 showed an increasing trend. There was significant difference between the 2 groups, as shown in fig. 4. Compared to the control group, the early apoptotic rate of osteoblasts in liraglutide intervention group is significantly lower $(\mathrm{P}<0.05)$ and the early apoptotic rate of osteoblasts was the lowest at the concentration of $10^{-8} \mathrm{M}$, as shown in fig. 5 . 


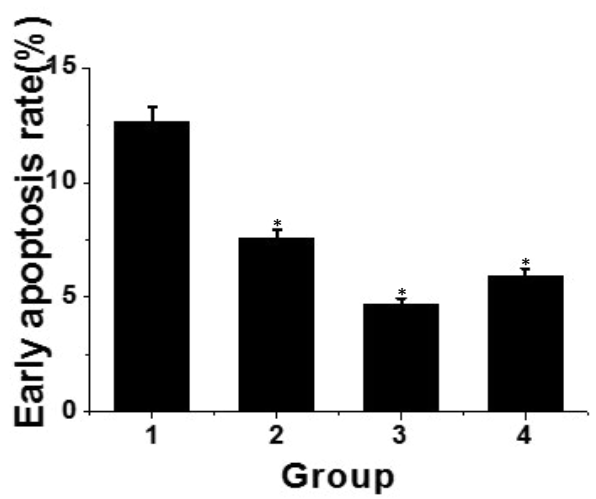

Fig. 5: Early apoptotic rate of osteocytes in each group Significantly different at $* \mathbf{P}<\mathbf{0 . 0 5}$

Osteoblast differentiation markers are GLP-1R, Runx2, OCN and ALP ${ }^{[16]}$. Runx2 is expressed in the early stage of wound healing. The expression is relatively high in the early stage and the expression location is in the nucleus, which is mainly distributed around the edge of the new bone trabecula, promoting differentiation in the early stage, and inhibiting differentiation in the late stage ${ }^{[17]}$. OCN is an osteoblast secreted protein, osteocalcin, also known as boney-carboxyglutamic acid-containing protein (BGP), is the main component of bone noncollagen protein and the specific protein of bone tissue. It is a hormone-like substance produced and secreted by osteoblasts. At present, serum OCN is considered to be a biochemical marker reflecting the function of osteoblasts $^{[18]}$. Alkaline phosphatase (ALP) is an early marker of osteogenesis. ALP is mainly distributed in Korean-bound transporter of cell membrane, which can promote cell maturation and calcification. Quantitative detection of ALP can reflect the differentiation level of osteoblasts. The higher the activity of ALP, the more obvious the differentiation of pre-osteoblasts into mature osteoblasts ${ }^{[19]}$. The high expression of ALP activity is an early marker of osteoblast differentiation and maturation. When ALP activity is increased, bone formation is enhanced and bone matrix mineralization is promoted. Therefore, the activity of ALP is a good indicator of osteoblast differentiation and functional status $^{[20]}$. GLP-1 plays its biological role by binding to GLP-1R. GLP-1R belongs to the glucagon receptor subfamily of 7 transmembrane $G$ protein-coupled receptor $B$ family. It is widely distributed in the pancreas, gastrointestinal tract, kidney, cardiovascular system, brain and bone and other tissues and organs. The combination of GLP-1 and GLP-1R has a positive effect on bone strength and quality ${ }^{[21]}$.

In this study, it is found that the OD value of liraglutide intervention group increases compared with the control group, indicating that the concentration of liraglutide in the study range of $10^{-9}-10^{-7} \mathrm{M}$ can promote the proliferation of osteoblasts. At the same time, the rate of proliferation of osteoblasts reached the highest when exposed to $10^{-8} \mathrm{M}$ liraglutide, which indicated that the at this concentration liraglutide has the strongest promoting effect on the proliferation of osteoblasts. Real-Time RT-PCR is used to detect the expression of Runx 2 and OCN. The expression of Runx 2 reached its peak on $\mathrm{d} 6$ while OCN reached on d 20. Liraglutide promoted ALP activity of MC3T3-E1 cells in a dosedependent manner during their differentiate into osteoblasts. GLP-1R exists in the cytoplasm and nucleus of MC3T3-E1 cells. Liraglutide increased the expression of GLP-1R in MC3T3-E1 cells in a dosedependent manner. GLP-1R existed in both cytoplasm and nucleus of MC3T3-E1 cells, and liraglutide can increase the expression of GLP-1R in MC3T3-E1 cells in a dose-dependent manner. After adding $10^{-7}$ $M$ liraglutide to the medium, the expression of GLP$1 \mathrm{R}$ increased maximally on $\mathrm{d} 12$. In conclusion, the above results indicated that liraglutide promoted the expression of mouse pre-osteoblast MC3T3-E1.

\section{Acknowledgements:}

This work was supported by The Scientific Research Fund of Ningxia Medical University (No.XZ2017012); Key Research project of Health and Family Planning Commission of Ningxia Hui Autonomous region (No. 2017- NW- 005).

\section{REFERENCES}

1. Marini F, Brandi ML. Genetic Determinants of Osteoporosis: Common Bases to Cardiovascular Diseases?. Int J Hypertens 2017;2010:360-73.

2. Chen X, Jia-Can SU. New focus on osteoporosis: differentiation fate of bone marrow-derived mesenchymal stem cells. Acad J Second Mil Med Univ 2017;38(4):397-404.

3. Saag K G, Petersen J, Brandi M L. Romosozumab or Alendronate for Fracture Prevention in Women with Osteoporosis. N Engl J Med 2017;377(15):1417.

4. Yoshimura N, Muraki S, Nakamura K. Epidemiology of the locomotive syndrome: The research on osteoarthritis/ osteoporosis against disability study 2005-2015. Jap J Rheumatol 2017;27(1):1-7.

5. Sessa G. Clinical guidelines for the prevention and treatment of osteoporosis from the Italian Society for Orthopaedics and Traumatology: preface. J Orthop Trauma 2017;(4):1-2.

6. Yabe D, Eto T, Shiramoto M. Effects ofDPP-4 inhibitor linagliptin andGLP-1 receptor agonist liraglutide on physiological response to hypoglycaemia in Japanese subjects with type 2 diabetes: A randomized, open-label, 2-arm parallel comparative, exploratory trial. Diabetes Obes Metab, 2017;19(3):442-47.

7. Gaballah HH, Zakaria SS, Mwafy SE. Mechanistic insights into the effects of quercetin and/or GLP-1 analogue liraglutide 
on high-fat diet/streptozotocin-induced type 2 diabetes in rats. Retour Au Numéro,2017;92:331.

8. Yuan Z, Li D, Feng P. A novel GLP-1/GIP dual agonist is more effective than liraglutideinreducinginflammationandenhancing GDNF release in the MPTP mouse model of Parkinson's disease. Eur J Pharmacol 2017;812:S0014299917304363.

9. Li J, Fu LZ, Liu L. Glucagon-Like Peptide-1 (GLP-1) Receptor Agonist Liraglutide Alters Bone Marrow Exosome-Mediated miRNA Signal Pathways in Ovariectomized Rats with Type 2 Diabetes. Med Sci Monit 2017;23:5410-19.

10. Ishii H, Niiya T, Ono Y. Improvement of quality of life through glycemic control by liraglutide, a GLP-1 analog, in insulinnaive patients with type 2 diabetes mellitus: the PAGE1 study. Diabetol Metab Syndr 2017;9(1):3.

11. Ke J, Liu Y, Yang J. Synergistic effects of metformin with liraglutide against endothelial dysfunction through GLP-1 receptor and PKA signalling pathway. Sci Rep, 2017; 7: 41085.

12. Dong W, Miao Y, Chen A. Delayed administration of the GLP-1 receptor agonist liraglutide improves metabolic and functional recovery after cerebral ischemia in rats. Neurosci Lett 2017;641:1-7.

13. Gupta G, Dahiya R, Dua K. Anticonvulsant effect of liraglutide, GLP-1 agonist by averting a change in GABA and brain glutathione level on picrotoxin-induced seizures. Excli J 2017;16:752.

14. Wang R F, Xue G F, Hölscher C. Post-treatment with the GLP1 analogue liraglutide alleviate chronic inflammation and mitochondrial stress induced by Status epilepticus. Epilepsy Res 2018;142:45.

15. Nonogaki K, Kaji T. Liraglutide. A GLP-1 Receptor Agonist, Which Decreases Hypothalamic 5-HT2A Receptor Expression, Reduces Appetite and Body Weight Independently of Serotonin Synthesis in Mice J Diabetes Res2018:1-6.

16. Wittbrodt E T, Eudicone J M, Farahbakhshian S. Comparison of low-dose liraglutide use versus other GLP-1 receptor agonists in patients without type 2 diabetes. Am J Manag Care 2018;24(8):S156.

17. Liou JH, Liu YM, Chen CH. Management of Diabetes Mellitus with Glucagon-like Peptide-1 (GLP-1) agonist liraglutide in renal transplant recipients: A retrospective study. Transplant Proc 2018;50(8):2502-05.

18. Han D, Chen W, Gu X. Cytoprotective effect of chlorogenic acid against hydrogen peroxide-induced oxidative stress in MC3T3-E1 cells through PI3K/Akt-mediated Nrf2/HO-1 signaling pathway. Oncotarget 2017;8(9):14680.

19. Yan X, Wu H, Wu Z. The New Synthetic H2S-Releasing SDSS Protects MC3T3-E1 Osteoblasts against H2O2-Induced Apoptosis by Suppressing Oxidative Stress, Inhibiting MAPKs, and Activating the PI3K/Akt Pathway. Front Pharmacol 2017;08(e48872):07.

20. Xu D, Gao Y, Hu N. miR-365 Ameliorates DexamethasoneInduced Suppression of Osteogenesis in MC3T3-E1 Cells by Targeting HDAC4. Int J Mol Sci 2017;18(5):977.

21. Sun X, Yang X, Zhao Y. Effects of $17 \beta$-Estradiol on Mitophagy in the Murine MC3T3-E1 Osteoblast Cell Line is Mediated via G Protein-Coupled Estrogen Receptor and the ERK1/2 Signaling Pathway. Med Sci Monit International Medical Journal of Experimental \& Clinical Research 2018;24:903-11.

This is an open access article distributed under the terms of the Creative Commons Attribution-NonCommercial-ShareAlike 3.0 License, which allows others to remix, tweak, and build upon the work non-commercially, as long as the author is credited and the new creations are licensed under the identical terms

This article was originally published in Special
issue on "Trends in therapeutic Management of
Various Conditions" Indian J Pharm Sci 2020:82(3)
spl issue 6;70-76

